\title{
CYANOPHYCEAN ALGAE FROM THE FOOTHILLS OF INDO-WESTERN HIMALAYA
}

\author{
Sunil Kumar Shukla, P.K. Misra* and Chandra Prakash Shukla \\ Phycology Research Laboratory \\ Department of Botany \\ University of Lucknow, Lucknow - 226007, India \\ E-mail: misrapkm@yahoo.com; shuklasks_algae@yahoo.com
}

\begin{abstract}
Twenty six cyanophycean taxa were collected from Pilibheet, Nainital foothills of western Himalayas, belong to 8 genera viz. Chroococcus, Aphanocapsa, Merismopedia, Oscillatoria, Lyngbya, Anabaena, Aulosira and Anabaenopsis. These taxa are represented by 22 species, 3 varieties and 1 forma. All these taxa from Pilibheet are the first report.
\end{abstract}

Key words: Cyanophycean algae, Pilibheet, Nainital.

\section{INTRODUCTION}

Earlier works of Singh (1959, 1961), Talpasayi (1962), Kamat (1968, 1973), Gupta and Nair (1962), Darbal et al. (1978), Khan (1970), Prasad and Mehrotra (1977), Habib (1999) and Seth et al. (2006) on fresh-water algae of western Himalayas reveal that a large part of this region is still unexplored. Singh and Chaturvedi (1970), Chaturvedi and Pandey (1976), Pandey and Chaturvedi (1979) and Pandey (1982) have described fresh-water algae of Rohilkhand division. Habib et al. (1998) have reported chlorococcales from Kotdwar. Habib and Chaturvedi (1999, 2000, 2001) have described chlorococcales and chaetophorales from Ramnagar, Ranikhet and Kumaon region, respectively. Misra et al. (2007) have described desmids from Kumaon Himalaya. Shukla et al.
(2007) have described chlorococcalean algae from Indo-western Himalaya. But, till now cyanophycean algae have not been explored from foothills of western Himalayas.

\section{MATERIALS AND METHODS}

Random sampling technique has been applied in the algal collection procedure. Sample collections were made from Pilibheet, Lalkuan and Kathgodam between November 2003 to January 2006 from different aquatic habitats by using planktonic mesh net. The algal samples were preserved in $4 \%$ formalin and slides were prepared by staning cyanophycean algae by methylene blue and mounted in glycerine. Detail studies were made by examining specimens under a Nikon Labophot microscope E-400 with H-III photomicrographic attachment. Species 
identification had been done after the Desikachary (1959) and Prasad and Srivastava (1992).

\section{RESULTS AND DISCUSSION}

Twenty six taxa, represented with 8 genera have been reported from this area. Genus Oscillatoria occurs dominantly in various lotic environments. Genera Chroococcus, Aphanocapsa and Anabaenopsis are in rare form. Genus Aulosira is also in rare form and reported from district Pilibheet. Morphotaxonomic description of all the taxa are as follows:

\section{Order - Chroococcales \\ Family - Chroococcaceae}

Chroococcus schizodermatics West (Pl. 1, fig. 1), Desikachary, T.V. (1959), (Pl. 26, fig. 17, Pp. 103)

Cell in-group of 2-4, blue green without sheath, 12-15 $\mu \mathrm{m}$ in diameter with sheath, 50-52 $\mu \mathrm{m}$ in diameter, sheath yellow to brown, very distinct lamellated.

Collection Number and Date: SKS - UTFH 15, 29/10/05

Locality: Chatta Tal, Pilibheet

Aphanocapsa biformis A. Br. (Pl. 1, fig. 2), Desikachary, T.V. (1959), (Pl. 21, fig. 4, Pp. 134)

Thallus olive green, gelatinous, expanded; cell 7.0 $\mu \mathrm{m}$ diameter, spherical, mostly with a spherical envelope, loosely arranged, 2-4 together in a common mucilaginous envelope, nannocytes about 2 diameter.

Collection Number and Date: SKS - UTFH 15, 29/10/05

Locality: Chatta Tal, Pilibheet.

Merismopedia glauca (Ehrenberg) Naegeli (Pl. 1, fig. 3), Prasad and Srivastava (1992), (Pl. 6 fig. 5 Pp. 44)

Cell 3-4 $\mu \mathrm{m}$, colony $20 \mu \mathrm{m}$ in diameter. Colony light blue green, almost rectangular with slightly

ECOPRINT VOL 16, 2009 sinuate-crenate margins cells; ovate or hemispherical, present in multiples of four, regularly arranged to form quadrangular colonies. Cell contents blue green homogenous, without granules but each cell has distinct centrally situated gas vacuole; cell wall smooth, thick.

Collection Number and Date: SKS - UTFH 5, $7 / 11 / 03$

Locality: Gola River, Lalkuan.

\section{Order - Nostocales}

Family - Nostocaceae

Oscillatoria calcuttensis Biswas (Pl. 1, fig. 5), Desikachary, T.V. (1959), (P1. 42, fig. 21, Pp. 237)

Trichome $2.0 \mu \mathrm{m}$ long, $14.0 \mathrm{um}$ broad. Thallus leathery brown; trichomes parallel straight, not constricted at the cross- walls, cross walls have 3 granules, blue-green, end cell conical, pointed, not capitate.

Collection Number and Date: SKS - UTFH 2, $7 / 11 / 03$

Locality: Gola River, Lalkuan

O. chalybea (Mertens) Gomont var. insulris Gardner (Pl. 1, fig. 9), Desikachary, T.V. (1959), (Pl. 38, fig. 18, Pp. 218)

Trichome $2.0 \mu \mathrm{m}$ long, $13.0 \mu \mathrm{m}$ broad. Thallus dark blue-green; trichome straight or lightly or irregularly spirally coiled, slightly constricted at the cross-walls, attenuated at the apex, and somewhat bent, septa not granulated, end cell obtuse, not capitate, without calyptra.

Collection Number and Date: SKS - UTFH 6, 10/11/03

Locality: Water logged crop field, Pantnagar.

O. chilkensis Biswas (Pl. 1, fig. 16), Desikachary, T.V. (1959), (Pl.39, fig. 1, Pp. 215)

Trichome $2.0 \mu \mathrm{m}$ long, $6.0 \mu \mathrm{m}$ broad. Trichomes somewhat curved, slightly constricted at the joints, 
apex of the trichomes very shortly tapering, very rarely undulated, not hooked; apical cell obtusely rounded, not pointed or capitate, calyptra none; cells shorter than the diameter, transverse walls not granulated, cell contents homogenous, pale bluegreen.

Collection Number and Date: SKS - UTFH 2, 7/11/03

Locality: Gola River, Lalkuan.

O. chlorina Kuetzing ex Gomont (Pl. 1, fig. 12), Prasad and Srivastava (1992), (Pl. 8 fig. 11 Pp. 61)

Trichome $2.0 \mu \mathrm{m}$ long, $10.0 \mu \mathrm{m}$ broad. Trichomes short solitary yellowish green somewhat straight not tapering at apex, unconstricted at the cross wall, cell contents yellowish -green homogenous without granules and gas-vacuoles; end cells rotund calyptra absent; cell wall thick, smooth.

Collection Number and Date: SKS - UTFH 15, 29/10/05

Locality: Chatta Tal, Pilibheet.

O. corallinae (Kutz.) Gomont (Pl. 1, fig. 13), Desikachary, T.V. (1959), (Pl. 40, fig. 16, Pp. 221)

Trichome $3.0 \mu \mathrm{m}$ long, $5.0 \mu \mathrm{m}$ broad. Trichomes constricted at the cross-walls, slightly bent or curved at the ends, a little attenuated; cells not granulated at the cross-walls; end cells slightly capitate, with a convex, slightly thickened membrane.

Collection Number and Date: SKS - UTFH 15, 29/10/05

Locality: Chatta Tal, Pilibheet.

O. cortiana Meneghini ex Gomont (Pl. 1, fig. 4), Desikachary, T.V. (1959), (Pl. 38, fig. 14, Pp. 233)

Trichome $3.0 \mu \mathrm{m}$ long, 5.0-7.0 $\mu \mathrm{m}$ broad. Thallus dull blue-green; trichome straight, slightly constricted at the joints, gradually tapering at the ends, bent, not capitate, blue-green; cells as long as broad or longer or shorter than broad, septa not granulated, end cell obtuse, without calyptra.

Collection Number and Date: SKS - UTFH 12, 27/05/04

Locality: Gola River, Kathgodam.

O. curviceps forma Rao (Pl. 2, fig. 3), Desikachary, T.V. (1959), (Pl. 39, fig. 10, Pp. 209)

Trichome $2.0 \mu \mathrm{m}$ long, 8.0-12.0 $\mu \mathrm{m}$ broad. Thallus light or dark blue-green; trichomes more or less straight, bent at the end or spirally coiled, not attenuated or very little attenuated, not constricted at the cross-walls, cross-walls sometimes granulated; end cells flat rounded, not capitate.

Collection Number and Date: SKS - UTFH 19, 29/10/05

Locality: Ghannai Tal, Pilibheet.

o. formosa Bory ex Gomont (P1. 1, fig. 6), Prasad and Srivastava (1992), (Pl. 5 fig. 6 Pp. 62)

Trichome $3.0 \mu \mathrm{m}$ long, $6.0 \mu \mathrm{m}$ broad. Trichomes elongated solitary or aggregated to form dark blue green masses, almost straight, very gently attenuated towards slightly bent apices, may or may not be constricted at the cross walls. All contents bright blue green homogenous without granules and gas vacuoles; end cells obtuse or bluntly rounded, non capitate, calyptra absent; cell wall thick, smooth.

Collection Number and Date: SKS - UTFH 14, 21/12/04

Locality: Kalsia Nala, Gola Bairaj, Kathgodam

O. laete - virens (Crouan) Gomont (Pl. 1, fig. 11), Prasad and Srivastava (1992), (Pl. 9 fig. 10 Pp. 64)

Trichome $2.0 \mu \mathrm{m}$ long, $3.0 \mu \mathrm{m}$ broad. Trichomes thin solitary or densely aggregated to form bright blue membranous stratum straight, fragile, gradually attenuated towards undulate or bent apex distinct, constricted at the cross walls. Cell 
contents blue green homogenous without granules and gas vacuoles; end cells more or less obtuse or somewhat pointed non-capitate, calyptra absent, cell wall smooth.

Collection Number and Date: SKS - UTFH 5, 7/11/03

Locality: Gola River, Lalkuan.

o. limosa Agardh ex Gomont (Pl. 1, fig. 15), Prasad and Srivastava (1992), (Pl. 9 fig. 4 Pp. 65)

Trichome $2.5 \mu \mathrm{m}$ long, $18.0 \mu \mathrm{m}$ broad. Trichomes solitary loosely or compactly entangled forming a dark blackish blue green or yellowish green thallus, almost straight not tapering towards apex may or may not be constricted at the cross walls. All contents blue green or olive green homogenous without gas vacuoles, granules may or may not be present, if present distributed regularly. End cells flatly rounded with or without slightly thick ended out membrane.

Collection Number and Date: SKS - UTFH 15, 29/10/05

Locality: Chatta Tal, Pilibheet.

O. okeni Agardh ex Gomont (Pl. 1, fig. 14), Desikachary, T.V. (1959), (Pl. 38, fig. 17, Pp. 231)

Trichome $2.5 \mu \mathrm{m}$ long, $7.0 \mu \mathrm{m}$ broad. Thallus dull blue-green; trichomes straight, fragile distinctly constricted at the cross-walls. End cells obtuse or sub conical not capitate, without calyptra.

Collection Number and Date: SKS - UTFH 6, $10 / 11 / 03$

Locality: Water logged crop field, Pantnagar.

O. ornata Kutz. ex Gomont (Pl. 2, fig. 2), Desikachary, T.V. (1959), (Pl. 37, fig. 12, Pp. 206)

Trichome $2.0 \mu \mathrm{m}$ long, $14.0 \mu \mathrm{m}$ broad. Thallus dark blue-green; trichome spirally coiled at the ends, constricted at the cross-walls, cross-wall granulated; apices slightly attenuated; end-cells rounded, not capitate, without thickened membrane.

Collection Number and Date: SKS - UTFH 2B, 10/11/03

Locality: Water logged crop field, Pantnagar.

O. princeps Vaucher ex Gomont (Pl. 2, fig. 1), Desikachary, T.V. (1959), (Pl. 37, fig. 14, Pp. 210)

Trichome $42 \mu \mathrm{m}$ broad, $5 \mu \mathrm{m}$ long. Trichomes blue-green, more or less brownish, violet or reddish, mostly forming a thallus, mostly straight, not constricted at the cross-walls, blue-green to dirty green, slightly or briefly attenuated at the apices and bent, end cells flatly rounded, slightly capitate without or with slightly thickened membrane.

Collection Number and Date: SKS - UTFH 15, 29/10/05

Locality: Chatta Tal, Pilibheet.

O. pseudogeminata $\mathrm{G}$. Schmed. var. unigranulata Biswas (Pl. 1, fig. 10), Prasad and Srivastava (1992), (Pl. 9 fig. 1 Pp. 70)

Trichome $2.5 \mu \mathrm{m}$ long, $3 \mu \mathrm{m}$ broad. Trichomes thin solitary or loosely entangled forming pale blue green thallus almost straight, not attenuated, not constricted at the cross walls. Cell contents olive green homogenous without gas vacuoles but a single rounded granule present in the middle on either side of the thick septa; end cells rounded, not capitate, calyptra absent; cell wall thick smooth.

Collection Number and Date: SKS - UTFH 6, 10/11/03

Locality: Water logged crop field, Pantnagar.

O. subbrevis Schmidle (Pl. 1, fig. 7), Desikachary, T.V. (1959), (Pl. 37, fig. 2, Pp. 207)

Trichome $2.0 \mu \mathrm{m}$ long, $12.0 \mu \mathrm{m}$ broad. Trichomes single, nearly straight, not attenuated at the apices; 
cells not granulated at the cross-walls; end-cell rounded, calyptra absent.

Collection Number and Date: SKS - UTFH 6, 10/11/03

Locality: Water logged crop field, Pantnagar.

o. tenuis Agardh ex Gomont (Pl. 1, fig. 8), Prasad and Srivastava (1992), (Pl.9 fig. 3 Pp. 7374)

Trichome $2.0 \mu \mathrm{m}$ long, $6.0 \mu \mathrm{m}$ broad. Trichomes thin solitary or aggregated to form olive green slimy masses straight or slightly curved, not attenuated, may or may not be constricted at the cross walls. Cell contents light blue green homogenous without gas vacuoles, granules may or may not be present, if present confined to septa; end cells rounded or hemispherical not capitate, calyptra absent; cell wall thick smooth.

Collection Number and Date: SKS - UTFH 2, 7/11/03

Locality: Gola River, Lalkuan.

Lyngbya birgei Smith (Pl. 2, fig. 6), Desikachary, T.V. (1959), (Pl. 50, fig. 7, Pp. 296)

Filament straight, seldom coiled, free floating, 3.0 $\mu \mathrm{m}$ broad, sheath firm, colorless, mostly unlamellated, seldom lamellated, trichome not constricted at the cross-walls, end rounded, not attenuated, not capitate, cells shorter than broad, $18.0 \mu \mathrm{m}$ long, sometimes with gas vacuoles.

Collection Number and Date: SKS - UTFH 6, 10/11/03

Locality: Water logged crop field, Pantnagar.

L. martensiana Menegh. ex Gomont var. calcarea Tilden (Pl. 2, fig. 5), Desikachary, T.V. (1959), (P1. 53, fig. 9, Pp. 319)

Thallus caespitose, expanded, penicillate, dull blue green, when dry sometimes violet, filaments curved or nearly straight, at the base intricate, above more or less parallel, sheath thin, papyraceous, colorless, colored violet by chlorzinc-iodide, trichomes $12.0 \mu \mathrm{m}$ broad, distinctly constricted at the cross-walls, cross-walls sometimes granulated not alternated, not capitate, blue- green; cell quadrate, end cell rounded.

Collection Number and Date: SKS - UTFH 6, $10 / 11 / 03$

Locality: Water logged crop field, Pantnagar.

L. putealis Mont. ex Gomont (Pl. 2, fig. 7), Desikachary, T.V. (1959), (Pl. 52, fig. 12 Pp. 318)

Thallus caespitose, penicillate dull blue green, when dry sometimes violet; filaments curved or nearly straight, at the base intricate more or less parallel; sheath thin, distinctly constricted at the cross walls, cross-walls sometimes granulated, not attenuated, not capitate, blue-green; cells quadrate of up to $8.0 \mu \mathrm{m}$ long, $10.0 \mu \mathrm{m}$ broad, end cell rounded.

Collection Number and Date: SKS - UTFH 15, 29/10/05

Locality: Chatta Tal, Pilibheet.

\section{Family - Nostocaceae}

Anabaena constricta (Szafer) Geitler (after Koppe), (Pl. 2, fig. 9), Desikachary, T.V. (1959), (Pl. 71, fig. 1, Pp. 394)

Trichome $4.0 \mu \mathrm{m}$ broad, Heterocyst $5.0 \mu \mathrm{m}$ broad. Trichomes single, not forming a thallus, with cells distinctly placed from one another, with brisk creeping movements with sheath; cells cylindrical and at the ends rounded or oval.

Collection Number and Date: SKS - UTFH 9, 21/12/04

Locality: Gola River, Lalkuan. 

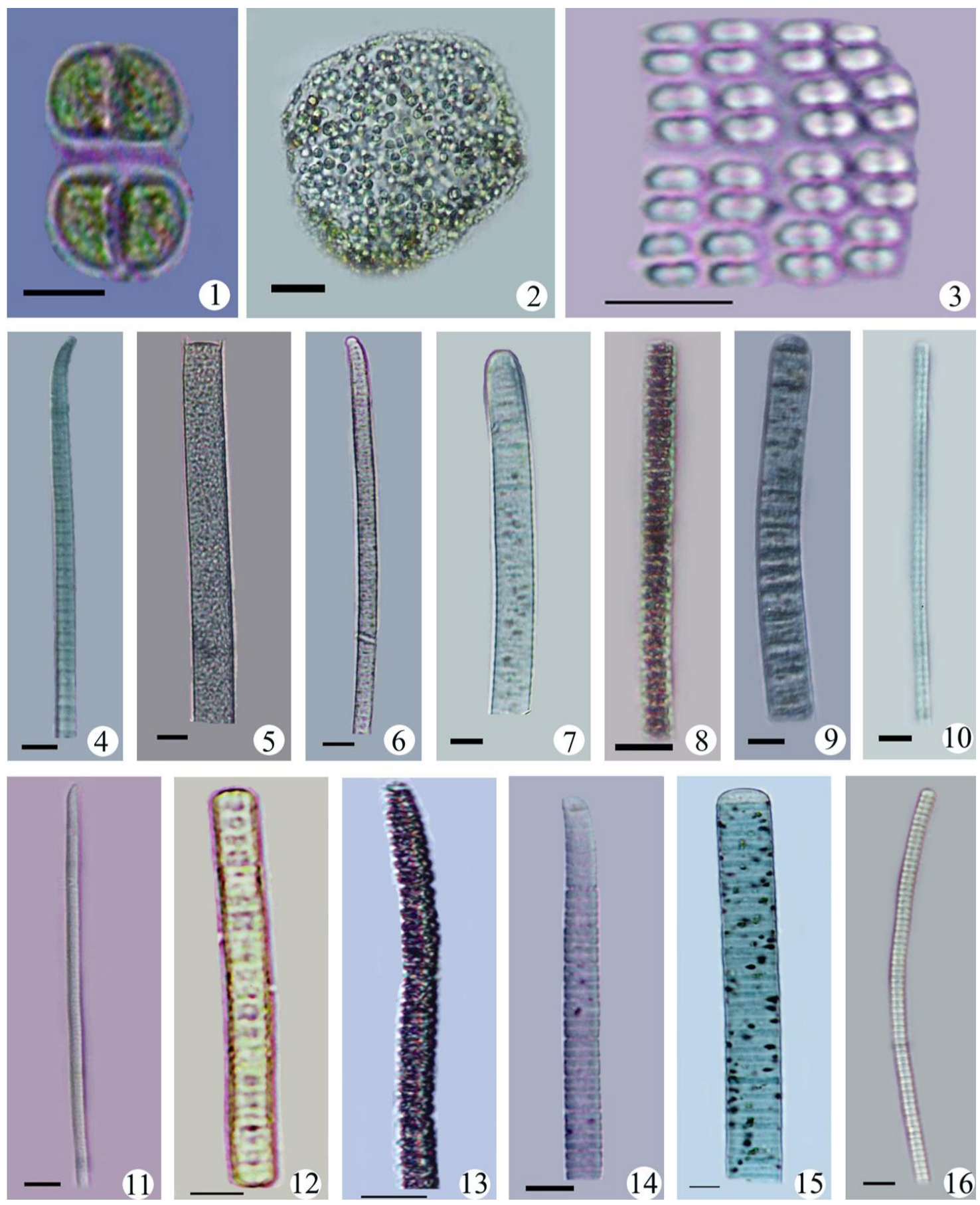

Plate 1. (Fig. 1. Chroococcus schizodermatics West, Fig. 2. Aphanocapsa biformis A.Br., Fig. 3. Merismopedia glauca (Ehr.) Naeg., Fig. 4. Oscillatoria cortiana Meneghini ex Gomont, Fig. 5. O. calcuttensis Biswas, Fig. 6. O. formosa Bory ex Gomont, Fig. 7. O. subbrevis Schmidle, Fig. 8. O. tenuis Ag ex Gomont, Fig. 9. O. chalybea (Mertens) Gomont var. insulris Gardner, Fig.10. O. pseudogeminata G. Schmed. var. unigranulata Biswas, Fig.11. O. laete - virens (Crouan) Gomont, Fig.12. O. chlorina Kuetz. ex Gomont, Fig.13. O. corallinae (Kuetz.) Gomont, Fig.14. O. okeni Ag. ex Gomont, Fig.15. O. limosa Ag. ex Gomont, Fig.16. O. chilkensis Biswas). 

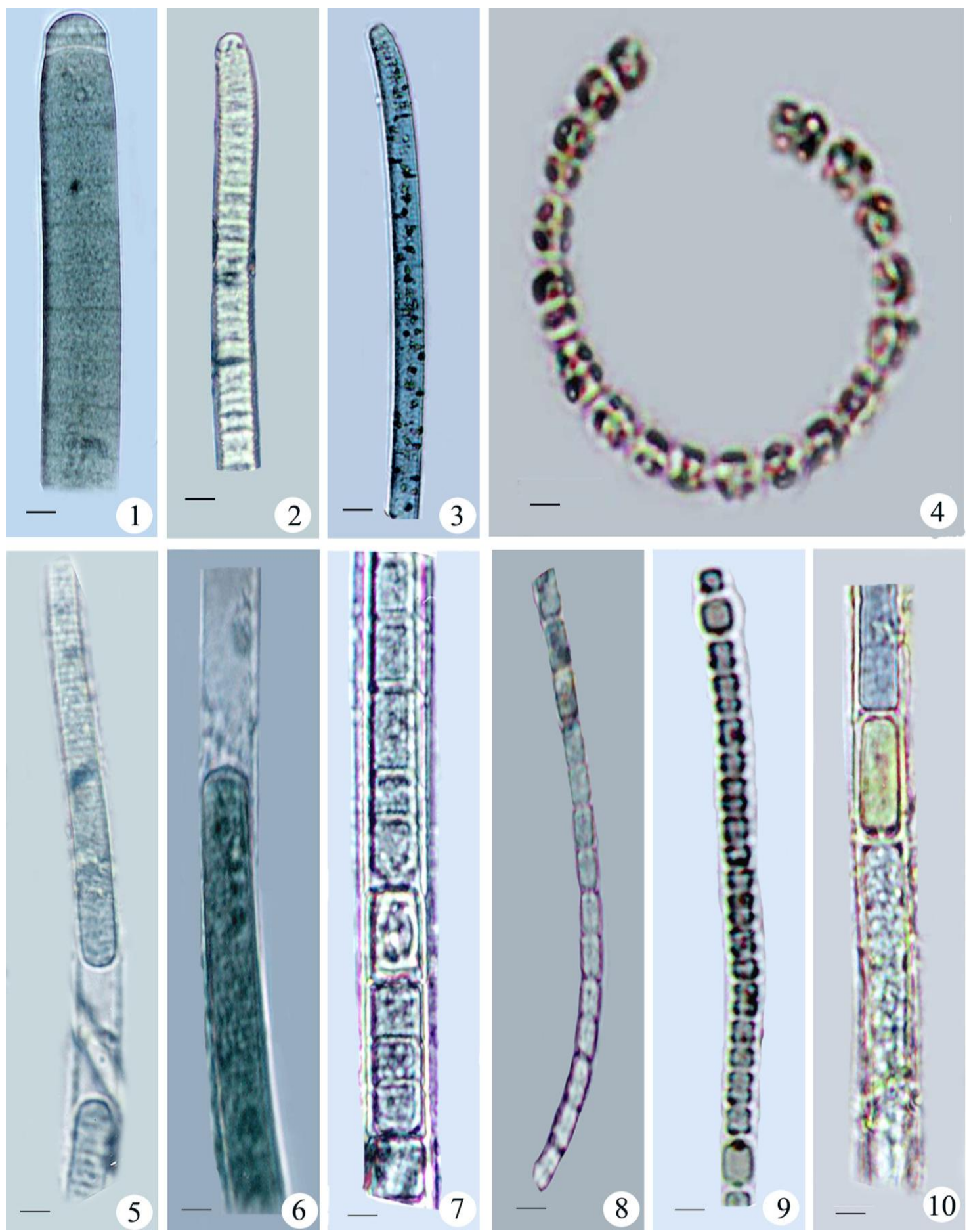

Plate 2. (Fig. 1. O. princeps Vaucher ex Gomont, Fig. 2. O. ornata Kutzing ex Gomont, Fig. 3. $O$. curviceps forma Rao, Fig. 4. Anabaenopsis circularis (G.S. West) Wolosz. and Miller var. javanica Wolosz. , Fig. 5. L. martensiana Menegh. ex Gomont var. calcarea Tilden, Fig. 6. Lyngbya birgei Smith, Fig. 7. L. putealis Mont. ex Gomont, Fig. 8. A. fuellabornii Schmidle, Fig. 9. Anabaena constricta (Szafer) Geitler (after Koppe), Fig. 10. Aulosira fertilissima Ghose var. tenuis Rao). 
A. fuellabornii Schmidle (Pl. 2, fig. 8), Desikachary, T.V. (1959), (Pl. 75, fig. 3, Pg 401)

Trichomes forming a small floccose, mucilaginous, blue-green mass, coiled or straight,sheath diffluent, seldom single; cell cylindrical with rounded ends, $5.0 \mu \mathrm{m}$ broad, mostly somewhat longer than board, end cell rounded, heterocysts barrel-shaped or cylindrical with rounded ends, spores or side of heterocyst, single or in pairs, ellipsoidal or cylindrical.

Collection Number and Date: SKS - UTFH 15, 29/10/05

Locality: Chatta Tal, Pilibheet.

Anabaenopsis circularis (G.S. West) Wolosz. et Miller var. javanica Wolosz. (Pl. 2, fig. 4), Desikachary, T.V. (1959), (Pl. 63, figs. 3 and 5, Pp. 354)

Trichomes free - swimming, very short, mostly spirally coiled, with $1-11 / 2$ spring, very seldom straight, $4.0 \mu \mathrm{m}$ broad, cells spherical or somewhat longer than broad, with a large granule.

Collection Number and Date: SKS - UTFH 15, 29/10/05

Locality: Chatta Tal, Pilibheet.

Aulosira fertilissima Ghose var. tenuis Rao (Pl. 2, fig. 10), Desikachary, T.V. (1959), (Pl. 80, fig. 3, Pp. 431)

Trichome $10.0 \mu \mathrm{m}$ broad, $2.0 \mu \mathrm{m}$ broad. Stratum expanded, dark blue-green, membranous; trichomes straight or a little flexuous, parallel or densely intricate, rarely with very short pseudobranches; cylindrical when young, later barrel-shaped, contents granular; sheath thick, at first gelatinous and hyaline, later firm and brown; heterocysts intercalary, oblong or elliptical, 10.0 $\mu \mathrm{m}$ broad and $22.0 \mu \mathrm{m}$ long; spores in series usually alternating with dead cell, generally oblong-elliptical, sometimes angular.

Collection Number and Date: SKS - UTFH 15, 29/10/05

Locality: Chatta Tal, Pilibheet.

\section{ACKNOWLEDGEMENTS}

Authors are thankful to Ministry of Environment and Forest, New Delhi for financial assistance and to the Head, Department of Botany, University of Lucknow, Lucknow for necessary laboratory facilities. CPS is thankful to C.S.I.R, New Delhi for financial assistance.

\section{REFERENCES}

Chaturvedi, U.K. and U.C. Pandey. 1976. A list of blue-green and green algae from Rohilkhand division, U.P., India-IV. Phycos, 15(1 and 2): 127-131.

Darbal, P.K., A.M. Bendre and L. Singh. 1978. Cyanophyceae of Rishikesh and its adjacent foothills. Botanical Progress 1:41-44.

Desikachary, T.V. 1959. Cyanophyta, A monograph, I.C.A.R., New Delhi.

Gupta, A.B. and G.U. Nair. 1962. A contribution to the algal-flora of Garhwal. J. Agra Univ. Res. Sci. 11(3):227-240.

Habib, I. and U.K. Chaturvedi. 1999. A systematic account of Chlorococcales from Ramnagar, Kumaon Himalaya. Phykos. 38(1 and 2):97-100.

Habib, I. and U.K. Chaturvedi. 2000. A systematic account of Chlorococccales from Kumaon Himalaya. Phykos 39(1 and 2):113118.

Habib, I. and U.K. Chaturvedi. 2001. Epiphytic algal flora of Kumaon Himalayas: Chaetophorales. Phykos $\mathbf{4 0}(1$ and 2):79-81.

Habib, I. 1999. Desmids of Shimla, Himachal Pradesh. Acta Botanica 24(1): 119.

Habib, I., J.O. Ghildiyal, C. Negi and U.K. Chaturvedi. 1998. A systematic account of Chlorococcales from Kotdwar, Garhwal. Phykos 37(1 and 2):125-129. 
Kamat, N.D. 1968. Algae of Shimla (Miscellaneous Note). J. Bombay Nat. Hist. Soc. 65(1):271-277.

Kamat, N.D. 1973. Algae of Nainital. J. Bombay Nat. Hist. Soc. 70(3):582-586.

Khan, M. 1970. Algal flora of Dehradun-I, Myxophyceae. Phycos. 9(2):126-131.

Misra, P.K., A.K. Rai and S.K. Shukla. 2007. Some fresh-water desmids from Kumaon Himalaya. J. Applied Biosciences 33(1):39-44.

Pandey, U.C. and U.K. Chaturvedi. 1979. Algae of Rohilkhand division, U.P., India-V. Phycos 18(1 and 2):37-43.

Pandey, U.C. 1982. Addition to the algal flora of Rohilkhand division VI, Cyanophyceae. Phycos 21:137-140.

Prasad, B.N. and M.N. Srivastava. 1992. Fresh water algal flora of Andaman and Nicobar Islands. Vol. I.B. Singh, Dehradun, India. 369 pp.
Prasad, B.N. and R.K. Mehrotra. 1977. Algal floristics in India - A Resume. Bull. Bot. Surv. India, 19(1-4):279-292.

Seth, K.P., M.K. Seth and P.K. Misra. 2006. A review of literature on algal flora of Himachal Pradesh. Phytotaxonomy 5:35-57.

Shukla, S.K., P.K. Misra and C.P. Shukla. 2007. Chlorococcalean Algae from the foothills of Indo-Western Himalaya. J. Indian Bot. Soc. (Accepted)

Singh, K.P. and U.K. Chaturvedi. 1970. Myxophyceae of the Rohilkhand division, U.P., India-II. Phycos. 9(1):36-40.

Singh, K.P. 1959. The myxophyceae of Kumaon Hills, U.P., India-I. Proceedings Indian Acad. Sci. 49:161-166.

Singh, K.P. 1961. Myxophyceae of Kumaon Hills, U.P., India-II. Uttar Bharti 8(1):101-108.

Talpasayi, E.R.S. 1962. The myxophyceae of the Kumaon Hills, U.P., India-II. Proc. Indian Acad. Sci. 55:251-252. 